\title{
The role of a foreign accent in the social and personal identification of a speaker
}

\author{
Marianna Popova* \\ Moscow State Linguistic University, Ostozhenka Str., 38 ,119034 Moscow, Russia
}

\begin{abstract}
The intensification of migration processes in Europe necessitates studying the foreign accent as a means of social and personal diagnostics within multicultural communities in the light of the increased number of cases of the "accent-based" discrimination, since the foreign accent is perceived negatively by native speakers and causes less confidence in a speaker. Studies carried out in recent decades suggest that external signs are gradually losing their leading role in social identification, giving way to more reliable and objective means pronunciation characteristics, since they are practically impossible to control. For this reason, an experimental phonetic research was carried out on the material of the German language in order to identify the nature of the influence of accentedness on the social identification of Russianspeaking immigrants from the position of German native speakers, as well as to determine the acceptation limits of accentedness degree of Russianspeaking bilinguals. With regard to the results of the study, it was found that a strong accent negatively affects the overall assessment of an individual in terms of the educational level, income and success of a speaker, while in terms of personal characteristics there is no pronounced negative attitude towards people speaking with a Russian accent, regardless of the degree of accentedness.
\end{abstract}

\section{Introduction}

At present, research in the field of contrastive phonetics within the contrastive linguistics is particularly topical. The increased interest of researchers in this area is explained by the urgency of studying the problems of intercultural communication in countries with active integration processes. The outstanding interest to researchers is the influence of the speaker's mother tongue in a foreign language speech on the perception and conditional "acceptation" of this person within the framework of a multicultural community.

The significance of research on auditory perception in the study of accent-colored speech cannot be overstated on account of the fact that it is the individual-subjective nature of the identification of a foreign language accent in the process of interpersonal communication that can prevail over the assessment of external characteristics $[1,2]$. Since external signs are gradually losing their significance in social diagnostics, speech and, in particular, accent become one of the most important means of social identification,

\footnotetext{
${ }^{*}$ Corresponding author: neunerin@gmail.com
} 
allowing to obtain information about the origin, social status, education level, intellectual development, self-identification, along with other characteristics of a speaker [3, 4]. In a communicative environment where a foreign language acts as a lingua franca, and social or ethnic categorization is difficult, "general mechanisms" for assessing accent-colored speech are activated, in which the main factors of assessment are fluency and clarity, along with "general accent stereotypes" $[5,6]$. It should be noted that regardless of the severity of the accent, accent-colored speech inspires less confidence and is evaluated by both native and non-native speakers more negatively than speech with near-native pronunciation $[7,8]$. And regardless of the fact that many multicultural states, in particular Germany, pursue an active "policy of tolerance" towards representatives of various ethnic groups, a strongly pronounced foreign accent contributes, according to the research results, to the activation of "passive tolerance", which has a detrimental effect on the prospects for the integration of immigrants into the host community and may even cause discrimination [9, 10]. Considering the fact that today Germany is the leader among the EU members in terms of the number of migrants hosted, and in 2018-2019, EU residents named immigration as the major problem, giving it more importance than taxes, pensions, unemployment rates and even terrorism, it seems critical to study interdependence of a speaker's pronunciation features with the formation of their social portrait, which can play a leading role in the process of subsequent integration into the host society, since the pronunciation characteristics are the "calling card" of a speaker. The Russian-speaking community in Germany is the third largest group of immigrants after the ones from Poland and Turkey and numbers, according to various sources, from 2 up to 3.5 million people [11], however until now, no studies have been conducted to establish the above relationship on the example of the Russian and German languages.

To determine the role of a foreign accent regarding social and personal diagnostics and social portraiture, an experimental phonetic study was carried out based on the material of the German language on the basis of the Moscow State Linguistic University in 2020. The results of this study allow setting the acceptance limits of the accentedness degree in relation to the social and personal characteristics of Russian immigrants, which can be used to make a conclusion about the actual attitude of the German-speaking host community to one of the largest immigrant communities.

\section{Materials and methods}

A corpus of phonograms in German with a total duration of more than 50 hours, compiled in the course of preparation was the material for the study. To test our hypotheses, we used the method of perceptual-auditory analysis, which is based on the works of the founder of the scientific school of fundamental and applied speech studies, R.K. Potapova, and involves conducting a series of perceptual-auditory studies on authentic language material with the assistance of test groups and auditors.

The aim of the study was to establish the dependence of the perception of German speakers about the personal and social characteristics of Russian-speaking tested individuals on the degree of accent coloring of their speech.

The study objectives were formulated as follows:

1) to establish how non-linguistically trained native German speakers scale accent of Russian-speaking bilinguals;

2) to study out how the degree of accentedness of Russian-speaking tested individuals correlates with their personal and social characteristics in the understanding of Germanspeaking auditors.

The object of the study was the implementation of the Russian accent in Germanlanguage communication with regard to the phonetic-phonological level. 
The subject of the study was the influence of a foreign accent on the speaker's social identification by a native speaker.

The formation of the auditors groups was carried out taking into account the following criteria: adult non-linguistically trained native German speakers without "migration background" were selected.

The study was carried out in 2 stages.

During the first stage, the auditors were asked to listen to the recordings of Russianspeaking "narrators" reading fragments of classical German literature and to evaluate the "narrators" accents according to the accentedness assessment scale, established by analogy to the classification developed by the American Council for the Teaching of Foreign Languages, and the model of accent gradation developed by Sh. Shaden (Table 1). The data on the tested individuals were coded with numbers. The duration of each phonogram was on average one minute. The recordings were played in the order specified by us.

Table 1. Accentedness assessment scale.

\begin{tabular}{|c|c|}
\hline \begin{tabular}{|c|} 
extrem ausgeprägter \\
Akzent
\end{tabular} & $\begin{array}{c}\text { Der Akzent beeinträchtigt das Verständnis. Man muss mehrmals hinhören, } \\
\text { um den Sprechenden zu verstehen. }\end{array}$ \\
\hline ausgeprägter Akzent & $\begin{array}{l}\text { Das Gesagte ist im Großen und Ganzen klar für Personen, die daran } \\
\text { gewöhnt sind, mit Ausländern zu kommunizieren. Mehrere } \\
\text { Aussprachefehler führen zu einer hohen Anspannung beim Zuhörenden } \\
\text { und können Verständnisprobleme verursachen. }\end{array}$ \\
\hline $\begin{array}{l}\text { durchschnittlicher } \\
\text { Akzent }\end{array}$ & $\begin{array}{l}\text { Das Gesagte ist klar, aber einige Aussprachefehler können } \\
\text { Verständnisprobleme verursachen. }\end{array}$ \\
\hline leichter Akzent & Die Aussprache beeinträchtigt das Verständnis nicht. \\
\hline kein Akzent & Die Person spricht akzentfrei. \\
\hline
\end{tabular}

In the first stage of the study, on the basis of an online questionnaire, 25 people (15 women and $10 \mathrm{men}$ ), aged 18 to 70 years, citizens without "migration background" from 9 federal states, were involved. Based on the data obtained as a result of the analysis, phonograms were selected, the estimates of which were the most unequivocal. In total, 8 phonograms were selected, which were formed into 4 groups according to the severity of the accent: the first group consisted of phonograms of the tested individuals, whose oralspeech discourse was assessed by the auditors as speech with a strong accent; the second group included phonograms of tested individuals with a medium accent; the tested individuals of the third group spoke, according to the majority of auditors, with a light accent, and the fourth group - with no accent. It was also decided to combine into one category the groups "very strong accent" (extrem ausgeprägter Akzent) and "strong accent" (ausgeprägter Akzent) for the next stage with a general name "strong accent", since it is difficult for German speakers to catch the difference between these categories.

The methodological basis for the second stage of the study was the research of $E$. Brennan and J. Brennan, dedicated to the study of attitudes towards Mexican Americans from the standpoint of the degree of accentedness. The auditors were asked to listen to 8 phonograms and evaluate the "narrators" according to social and personal characteristics, based on the proposed oppositions, on a scale from 0 to 10 , where 0 is the lowest score, 10 is the highest one, and 5 is "I cannot decide" (Fig. 1). The data on the tested individuals were coded with numbers. The text content was not taken into account in the assessment. 


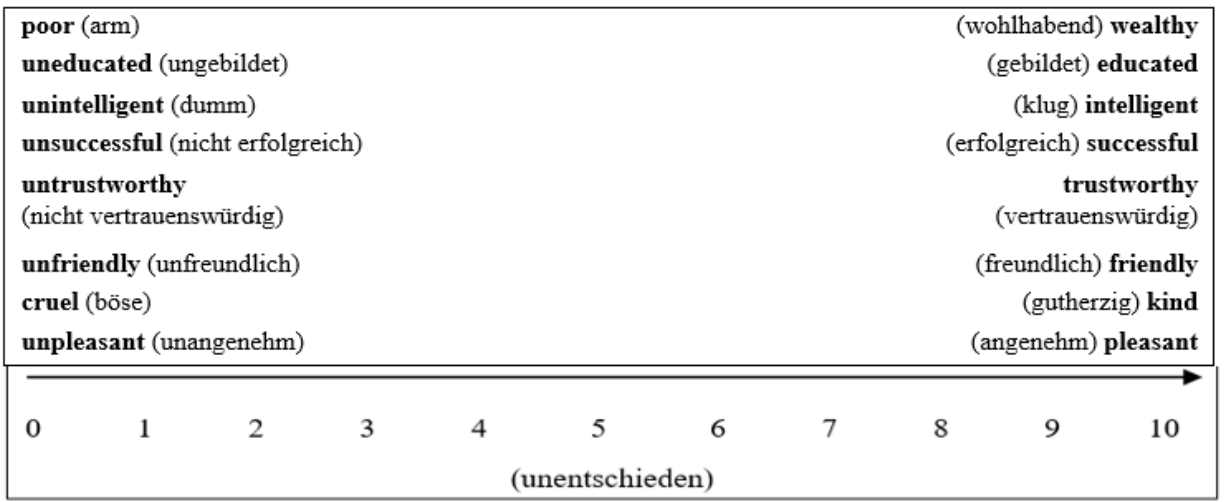

Fig. 1. The scale for assessing the social and personal characteristics of Russian-speaking "narrators" by German-speaking auditors.

On repeated listening, the auditors were asked to answer the following questions:

1. How likely is it on a scale from 0 to 10 , where 0 - "under no circumstances", 10 "most probably", 5 - "I cannot decide" that you would let a houseroom to this person, supposing that according to other parameters they are not inferior to other candidates?

2. In which three of the proposed professions do you see this person?

There were 9 options for gender-neutral job titles to choose from, taking into account the social status: high, medium or low:

1) High social status: company manager (Unternehmensleiter*in), doctor (Arzt/Ärztin) and lawyer (Rechtsanwalt*in);

2) Average social status: teacher (Lehrkraft), nursery school teacher (Erzieher*in), hairdresser (Friseur*in);

3) Low social status: dustman (Müllabfuhr), cleaning worker (Reinigungskraft), cashier (Kassier*in).

Positions were presented without reference to the social status.

\section{Results and discussion}

In the study by E. Brennan and J. Brennan, the oppositions were combined into two factors using factor analysis. The first four oppositions entered the "status" factor, and the next the "solidarity" factor. Since this study contains additional questions about the probability of letting a houseroom and the social aspect of the job titles associated with the "narrators", we considered it appropriate to reproduce the factor analysis (Table 2), the results of which revealed that the most significant are two factors. The first factor loads questions 1-4, and the second one burdens questions 5-8. For the convenience of interpreting the data, we named the first one as the "social characteristics" factor, and the second one - the "personal characteristics" factor.

Table 2. Factor matrix of assessments of social and personal characteristics of Russian-speaking tested individuals.

\begin{tabular}{|c|c|c|c|c|c|c|c|c|c|c|}
\hline Question No. & 1 & 2 & 3 & 4 & 5 & 6 & 7 & 8 & 9 & 10 \\
\hline Factor 1 & 0.87 & 0.86 & 0.78 & 0.85 & & & & & 0.51 & 0.75 \\
\hline Factor 2 & & & & & 0.76 & 0.88 & 0.87 & 0.78 & 0.64 & \\
\hline
\end{tabular}


It should be noted that the factor of "social characteristics" also included questions about the probability of letting a houseroom and the social aspect of the job titles associated with the "narrators". In its turn, question No. 9 has loads on both factors. This indicates that the likelihood of letting a houseroom depends on both social and personal characteristics of the speaker.

Next, analysis of variance (ANOVA) was carried out aimed at identifying significant differences in the assessment by the German speakers of each of the tested individuals, taking into account the assessment of their pronunciation characteristics by the auditors (Table 3).

Table 3. Analysis of variance results.

\begin{tabular}{|c|c|c|}
\hline Indicator values & F-criterion & p-value \\
\hline Poor - wealthy & 44.73 & $0.00^{* *}$ \\
\hline Uneducated - educated & 35.26 & $0.00^{* *}$ \\
\hline unintelligent - intelligent & 24.09 & $0.00^{* *}$ \\
\hline Unsuccessful - successful & 39.89 & $0.00^{* *}$ \\
\hline Untrustworthy - trustworthy & 7.98 & $0.00^{* *}$ \\
\hline Unfriendly - friendly & 5.64 & $0.00^{* *}$ \\
\hline Cruel - kind & 3.34 & $0.00^{* *}$ \\
\hline Unpleasant - pleasant & 15.31 & $0.00^{* *}$ \\
\hline Probability of renting a house & 13.66 & $0.00^{* *}$ \\
\hline Social status of the profession & 67.09 & $0.00^{* *}$ \\
\hline
\end{tabular}

Note. ${ }^{* *} \mathrm{p}<0.01$

Since all the coefficients are statistically significant, we can conclude that the speaker's articulatory characteristics affect the assessments of their personal and social characteristics, the social status of the professions associated with them, as well as the willingness of German-speaking auditors to let them a houseroom.

Further pairwise differences for each of the set scales were examined.

On the basis of the obtained data, within the framework of the opposition "uneducated educated" (Fig. 2), tested individual No. 3 and No. 6 were rated significantly lower than the rest. Since they both show a strong accent, we can conclude that people with a strong accent are ranked lower on this opposition. Significantly lower scores of tested individual No. 2 can be explained, in our view, by individual features of pronunciation, personal preferences of the auditors and the order of presentation of phonograms. 


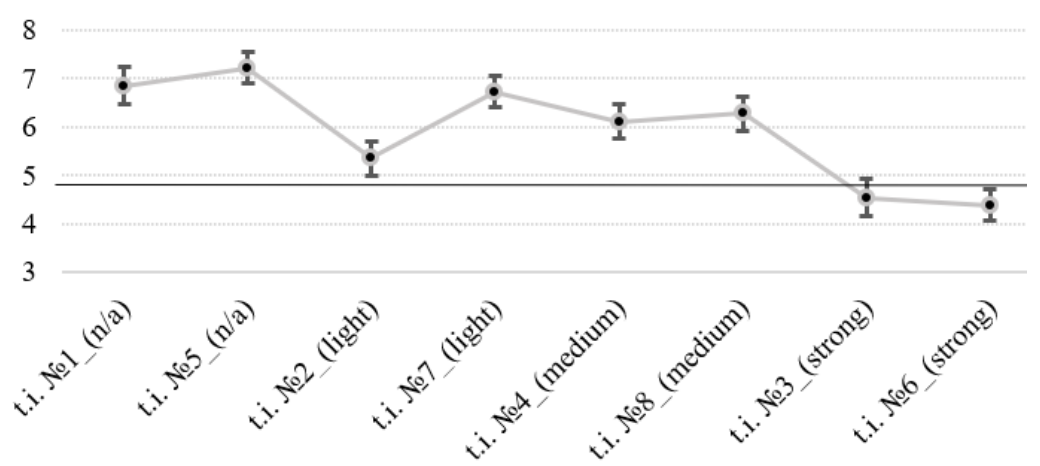

Fig. 2. Average estimates of tested individuals by native German auditors according to the "uneducated - educated" scale (confidence interval - 95\%)

Where: $\mathrm{n} / \mathrm{a}-$ no accent

For the rest of the social oppositions "poor - wealthy", "unintelligent - intelligent" and "unsuccessful - successful", identical reactions were obtained, as in the answer to the question about the social status of the professions associated with the tested individuals.

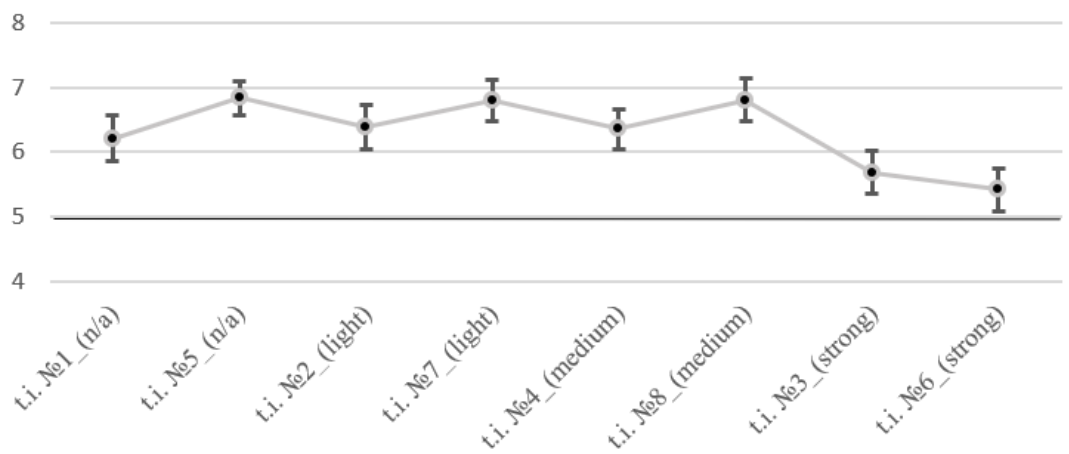

Fig. 3. Average estimates of tested individuals by native German auditors according to the "untrustworthy - trustworthy" scale (confidence interval - 95\%)

Where: $\mathrm{n} / \mathrm{a}-$ no accent

As we can see in graph 3 (Fig. 3), tested individuals with a strong accent were rated significantly lower in the opposition "untrustworthy - trustworthy", while the estimates of tested individuals with near-native pronunciation as well as with a light or medium accent do not differ significantly from each other. We can observe a similar picture for the opposition "unpleasant - pleasant", as well as when answering the question about the probability of letting a houseroom.

It should be noted that within the opposition "cruel - kind" (Fig. 4), as well as in the opposition "unfriendly - friendly", the absence of significantly lower ratings in tested individuals with a strong accent is characteristic. 


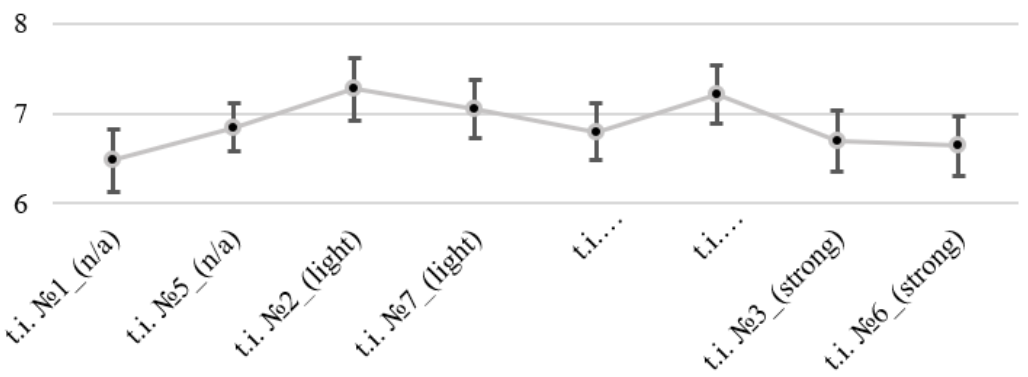

Fig. 4. Average estimates of tested individual by native German auditors according to the "cruel kind" scale (confidence interval - 95\%)

Where: $\mathrm{n} / \mathrm{a}-$ no accent

The tendencies observed in the auditors' assessments of the social characteristics of the tested individuals are also traced in the answers to the question about the probability of letting a houseroom to the tested individuals provided that they are not inferior to other candidates in other parameters (Fig. 5). Tested individuals with a strong accent received significantly lower estimates (barely exceeding a neutral value $=5$ ) than all other tested individuals. This suggests that a strong accent can act as a barrier in finding housing for candidates with a strong accent. Besides we can see that for the rest of the groups of tested individuals, the results do not have statistically significant differences. The exception is tested individual No. 2, who was assessed more negatively on all four social characteristics earlier - their score is significantly lower than that of tested individuals No. 1 and No. 5 (no accent), No. 7 (light accent) and No. 8 (medium accent).

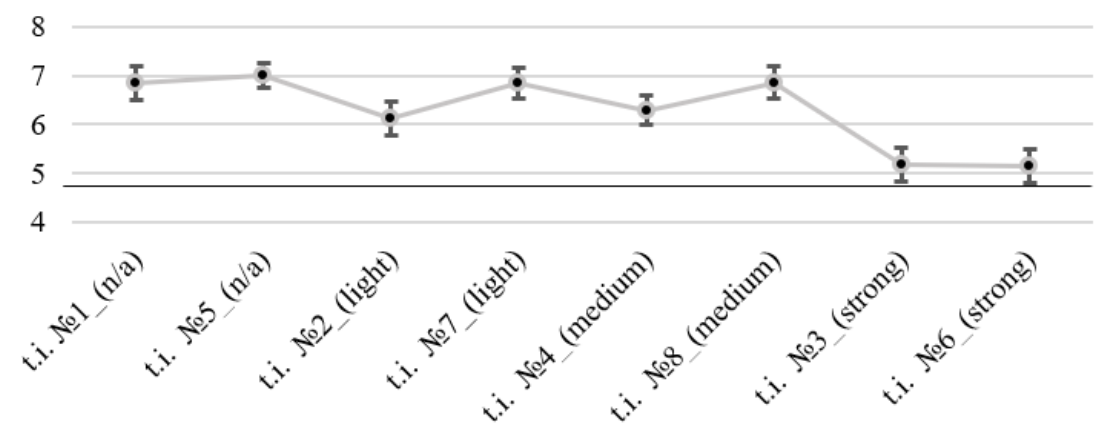

Fig. 5. The average scores of the tested individuals as per the scale "under no circumstances - most probably" when answering the question about the probability of letting this person a houseroom, provided that in other parameters they are not inferior to other candidates (confidence interval $95 \%)$.

Where: $\mathrm{n} / \mathrm{a}-$ no accent

The last question did not have a rating scale, that is why each profession was assigned a certain coefficient: professions of low social status (cleaning worker, cashier, dustman) - 1 , professions of medium social status (hairdresser, nursery school teacher, teacher) - 3, professions of high social status (company manager, doctor, lawyer) -5 . According to the analysis of variance (ANOVA) results (Fig. 6), statistically significant differences are observed only in the case of assessing both tested individuals with a strong accent, and in tested individual No. 2, which coincides with the results of assessing social characteristics and with the answers to the question about the probability of letting a houseroom. Also a statistically significant difference between the estimates of tested individual demonstrating a lack of accent and those with a strong accent should be stressed. 


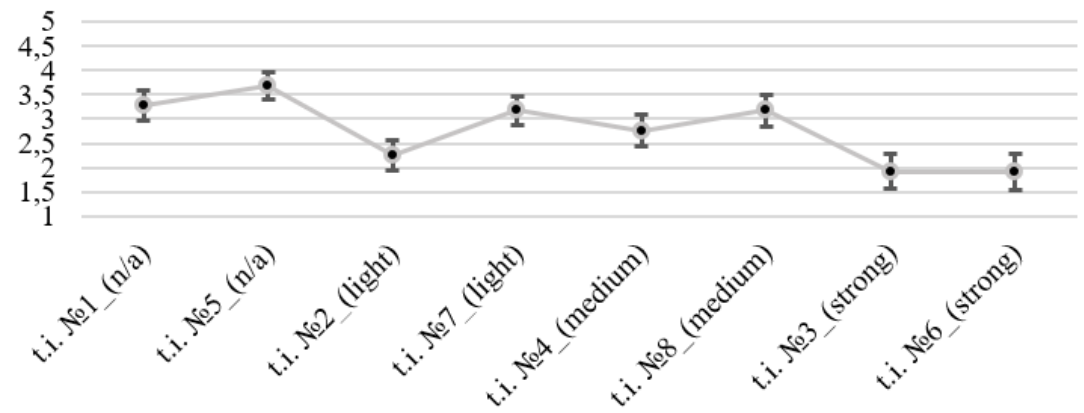

Fig. 6. Average estimates of tested individual by auditors-native German speakers when answering the question "In which three of the proposed professions do you see this person" (confidence interval $-95 \%)$.

Where: $\mathrm{n} / \mathrm{a}-$ no accent

\section{Conclusions}

1. The degree of severity of the Russian accent affects the assessment of the level of income, education, social success and intelligence capabilities of a speaker by native German speakers: tested individuals with a strong accent are generally rated significantly lower in these indicators than tested individuals with no accent, light or medium accent. Tested individuals who demonstrate a strong accent are generally rated significantly lower on social scales and they are regarded as rather untrustworthy by native German speakers.

2. Regarding personal characteristics, the results of the study indicate the absence of an expressed negative attitude towards people who speak with a Russian accent, regardless of its severity. The difference between the assessments of the personal characteristics of the tested individuals from the social ones can be the influence of upbringing, mentality and the "policy of tolerance" actively promoted in Germany. As a consequence civil solidarity can be observed in the positive assessment of the personalities of all tested individuals.

3. When correlating the tested individuals with job titles of low, medium and high social status, the factor of social characteristics plays the main role, whereas when deciding on the probability of letting a houseroom, native German speakers also rely on the factor of personal characteristics.

4. The assessments of auditors may also be influenced by their personal preferences and individual peculiarities of the tested individuals, but the general trends observed in our study bring us to the conclusion that, in general, they do not have a significant impact.

5. The negative influence of a strong accent on the overall assessment of a person in terms of the education level, income and success of a speaker is most clearly tracked: people with a strong accent received significantly lower estimates. In the present case, there is no significant difference in the assessment of tested individuals with a light, medium accent, as well as of the ones with near-native pronunciation.

6. The degree of accentedness has no impact on the assessment of some personal characteristics, in particular, kindness and friendliness.

7. The absence of a prominent ranking in the perception of the degrees of accentedness can be explained by the participation of German citizens in the study who are favorable to immigrants and by the influence of "socially approved opinion" and "passive tolerance" as a result of the multiculturalism policy carried out in Germany over the recent years, which is aimed at suppressing any manifestations of intolerance, xenophobia and racism. 


\section{References}

1. R.K. Potapova, V.V. Potapov, SPECOM, 8773 (2014)

2. R.K. Potapova, V.V. Potapov, SPECOM, 9811 (2016)

3. K.D. Kinzler, K. Shutts, J. Dejesus, E.S. Spelke, Social Cognition, 27 (2009)

4. T. Rakić, M.C. Steffens, A. Mummendey, JPSP, 100 (2011)

5. J.F. Dovidio, A. Gluszek, The handbook of intergroup communication (2012)

6. R. Mai, S. Hoffmann, J Consum Psychol, 24 (2014)

7. F. Krings, J. Olivares, IJP, 42 (2007)

8. J. Roessel, Ch. Schoel, R. Zimmermann, D. Stahlberg, J Lang Soc Psychol, 38 (2017)

9. U. Hinrichs, Multi Kulti Deutsch. Wie Migration die deutsche Sprache verändert (2013)

10. Deutsche Welle, https://www.dw.com/

11. Medien Dienst Integration, https://mediendienst-integration.de/ 